relevant contemporary data on this subject and provide an algorithm for the management of early PRSC.

Method A systematic review was undertaken in accordance with PRISMA guidelines. Studies published between 2000 and 2017 describing the clinical management of PRSC in patients with UC within 30 days of primary ileoanal pouch surgery were included. A qualitative analysis was undertaken due to the heterogeneity and quality of studies included.

Results 1157 abstracts and 266 full text articles were screened. Twelve studies were included for analysis involving a total of 207 patients. The studies described a range of techniques including image-guided, endoscopic, surgical and endocavitational vacuum methods. Based on the evidence from these studies, an algorithm was created to guide the management of early PRSC.

Conclusion Although the rate of successful salvage following early PRSC has improved over time there is a paucity of research correlating the method used with functional outcome. Short course Endo-SPONGE ${ }^{\circledR}$ therapy with early surgical closure seems to offer increased chance of salvage. We present an algorithm for the management of early PRSC.

\section{PTU-063 MISS RATES FOR COLORECTAL CANCER INVESTIGATED WITH COMPUTER TOMOGRAPHY SCANS}

Sorin Stanciu*, Nishchay Chandra. Royal Berkshire Hospital, Reading, UK

\subsection{6/gutjnl-2018-BSGAbstracts.404}

Introduction Computer tomography (CT) scans are often the initial investigation for patients suspected of colorectal cancer (CRC) because they are better tolerated than colonoscopy. This study aims to evaluate the CRC false negative results (or miss rates) for CT investigations of the colon.

Methods This is a retrospective review of 773 consecutive CRC cases encountered at Royal Berkshire Hospital between 2014 and 2016. Evidence of CT investigations in the previous 3 years was obtained from computerised health records. Only CT scans with the indication suggestive that it was done for suspected bowel malignancy were labelled as 'missed cancer'. CT scans done to investigate other abdominal organs were not considered. Statistical analysis was done with a confidence interval of $95 \%$.

Results $5.4 \%$ of patients diagnosed with CRC had an unremarkable CT scan in the previous 3 years. The indications included mostly iron deficiency anaemia, change in bowel habit, weight loss, abdominal pain and rectal bleeding. Patients being missed were significantly older than the rest of CRC patients (78.2 years vs. 69.5 years, $p=0.000003)$. A higher proportion of right sided cancers were missed as compared to left sided cancers $(7.3 \%$ vs. $4.8 \%)$ but this was not statistically significant $(p=0.36)$. Average time from scan to diagnosis was 512.9 days (1.4 years). Most CT studies (33 of 42, 79\%) were after administration of oral contrast. Two were CT colonographies that missed one caecal cancer and one rectal cancer at 1.2 and 1.8 years before diagnosis.

Concerning the entire cohort of CRC cases, mean age was 70, male:female ratio was 1:1.16, left sided lesions accounted for $60 \%$ and right sided lesions $34 \%$. All of these values are comparable to national statistics.

Conclusions Approximately 1 in 20 patients diagnosed with colorectal cancer had at least one CT scan with no evidence of bowel malignancy in the previous 3 years. Bowel cancer should not be easily excluded by an unremarkable CT scan if there is a high clinical suspicion, especially in a patient older than 70 , regardless of the type of scan.

\section{PTU-064 IMMUNE CHECKPOINT INHIBITOR COLITIS- A REVIEW OF CURRENT MANAGEMENT TRENDS}

${ }^{1}$ Mirashini Swaminathan*, ${ }^{2}$ Anna Olsson-Brown, ${ }^{2}$ Sreedhar Subramaniam, ${ }^{1}$ Mark Pritchard. ${ }^{1}$ Department of Cellular and Molecular Physiology, University of Liverpool, Liverpool, UK; 2University of Liverpool, Liverpool, UK

\subsection{6/gutjnl-2018-BSGAbstracts.405}

Introduction Immune checkpoint inhibitors (CPIs) are novel agents that work by boosting the body's immune system to fight tumour cells and are transforming cancer therapy. They are generally well tolerated but can cause side effects that mimic various autoimmune diseases. With its rising use across many tumour types, the prevalence of immune related adverse events such as colitis is fast becoming an issue encountered by many gastroenterologists. This review aims to characterise the current trends in management of CPI-induced colitis.

Methods An electronic database search was conducted on Pubmed and Embase. A total of 48 papers were identified for final analysis. This included 29 case reports and 19 case series describing the management of patients with CPI-induced colitis.

Results 48 papers containing 294 patients were included in the review. Of these, 264 were treated with CTLA- 4 inhibitors, 18 with PD-1 inhibitors, 1 with PDL-1 inhibitors and 10 with combination therapy. Majority of patients (196) received treatment for melanoma. Other malignancy types included non-small cell lung cancer, urothelial malignancy and prostate cancer. A total of 226 patients with CPI-induced colitis were treated with steroids (oral or intravenous). Of these $61 \%$ responded to steroids alone whilst $47 \%$ required further treatment with infliximab. $94 \%$ of patients treated with infliximab had resolution of colitis. 8 patients were treated with vedolizumab after steroid failure and all of these patients had resolution. 20 patients required surgery due to complications such as perforation or ischaemia, 3 had infliximab prior to surgery.

Patients required a median of 2 doses of infliximab to attain resolution of colitis and where follow up data was available, there was no symptom relapse post treatment with infliximab.

Conclusions This review highlights that a step wish approach similar to the management of inflammatory bowel disease should be used to manage patients with CPI-induced colitis. However there should be an early consideration for use of biologic therapy. Protocols including a multi-disciplinary approach should be developed to ensure that gastroenterologists are aware of treatment modalities should these patients present to them.

\section{PTU-065 SERUM LIPID PROFILE AND KRAS MUTATION ACCORDING TO TUMOUR LOCATION IN METASTATIC COLORECTAL CANCER}

1,2Maria Tabuso*, ${ }^{1,2}$ Ramesh Arasaradnam. 'University Hospitals Coventry and Warwickshire, Coventry, UK; ${ }^{2}$ Warwick University, UK

10.1136/gutjnl-2018-BSGAbstracts.406

Introduction In metastatic colorectal cancer mutated Kirstin rat sarcoma viral oncogene (KRAS) and tumour location are 